\title{
Suitability of 7 Aortic Stent-Graft Models for MRI- Based Surveillance
}

\author{
Maarten J. van der Laan, MD; Lambertus W. Bartels, PhD²; \\ Chris J.G. Bakker, PhD2; Max A. Viergever, PhD²; and Jan D. Blankensteijn, MD³
}

Departments of ${ }^{1}$ Vascular Surgery and ${ }^{2}$ Radiology/lmage Sciences Institute, University Medical Center Utrecht, The Netherlands. ${ }^{3}$ Department of Vascular Surgery, University Medical Center Nijmegen Radboud, Nijmegen, The Netherlands.

\begin{abstract}
$\checkmark$
Purpose: To evaluate the magnetic resonance imaging (MRI) characteristics of commercially available stent-grafts used for abdominal aortic aneurysm repair.

Methods: Seven endovascular grafts (AneuRx, Lifepath, Talent, Excluder, Zenith, Quantum LP, and Ancure) were suspended in a water bath containing gadolinium and scanned using a 1.5-T clinical MRI scanner. Two different scan techniques ( $T_{1}$-weighted spoiled gradient echo and spin echo) based upon a clinical MRI endograft surveillance protocol were used for each stent-graft. The scans were evaluated for susceptibility artifacts and radiofrequency (RF) shielding and caging artifacts.

Results: For most endografts, the lumen and structures surrounding the endograft were well visualized. However, the ferromagnetic properties of the Zenith and Lifepath devices resulted in large susceptibly artifacts that obliterated the endograft lumen as well as adjacent structures. All fully supported grafts showed some amount of signal loss from the graft lumen caused by RF caging. For the Ancure graft, evaluation around the attachment sites might be problematic.

Conclusions: An MRI-based surveillance protocol appears to be a viable option for the AneuRx, Talent, Excluder, and Quantum LP stent-grafts.
\end{abstract}

J Endovasc Ther 2004;11:366-371

Key words: abdominal aortic aneurysm, endovascular repair, stent-graft, surveillance, magnetic resonance imaging, artifacts

For adequate evaluation of treatment success after endovascular aneurysm repair, several specific items should be monitored: the effectiveness of aneurysm sac exclusion, graft patency, graft migration, and graft integrity. Several recent studies have shown magnetic resonance imaging (MRI) and magnetic resonance angiography (MRA) techniques to be more sensitive for endoleak detection than standard computed tomographic angiography (CTA). ${ }^{1-3}$ Indeed, MRI and MRA tech- niques are potentially well suited for the evaluation of aneurysm sac exclusion and graft patency. For morphological assessments, such as diameter or volume measurements, $\mathrm{MRI}$ and MRA have advantages over CTA as well. Because of the excellent soft tissue contrast, the aneurysm sac can be easily distinguished from the surrounding tissue and structures. However, in the evaluation of graft integrity and migration, neither MRI techniques nor CTA is ideal. For these purposes,

Address for correspondence and reprints: J.D. Blankensteijn, MD, University Medical Center Nijmegen Radboud, 410 Dept of Surgery, PO Box 9101, 6500 HB Nijmegen, The Netherlands. Fax: 31-24-3540501; E-mail: j.blankensteijn@chir.umcn.nl 
TABLE 1

Metal Components of the Endografts Used in This Study

\begin{tabular}{llcc}
\hline & Metal Support & Metal Attachment & Metal Markers \\
\hline AneuRx & Nitinol & Nitinol & Platinum \\
Lifepath & Elgiloy & Elgiloy & Elgiloy \\
Talent & Nitinol & Nitinol & Nitinol \\
Excluder & Nitinol & Nitinol & Gold \\
Zenith & Stainless steel & Stainless steel & Gold \\
Quantum LP & Nitinol & Nitinol & Tantalum \\
Ancure & None & Elgiloy & Platinum \\
\hline
\end{tabular}

Elgiloy is a cobalt/chromium alloy.

abdominal radiography seems to be superior. ${ }^{4,5}$

One possible disadvantage of MRI techniques is that the endografts have to be MRI compatible, so a major potential problem is the presence of metallic components in the endografts. Metallic structures can produce artifacts that may considerably degrade image quality. The purpose of this study was to investigate which commercially available endografts can be used in MRI-based surveillance after EVAR.

\section{METHODS}

\section{In Vitro Experiments}

Endografts were evaluated for artifacts that influenced the endograft surroundings and artifacts influencing the appreciation of the device lumen. Seven commercially available

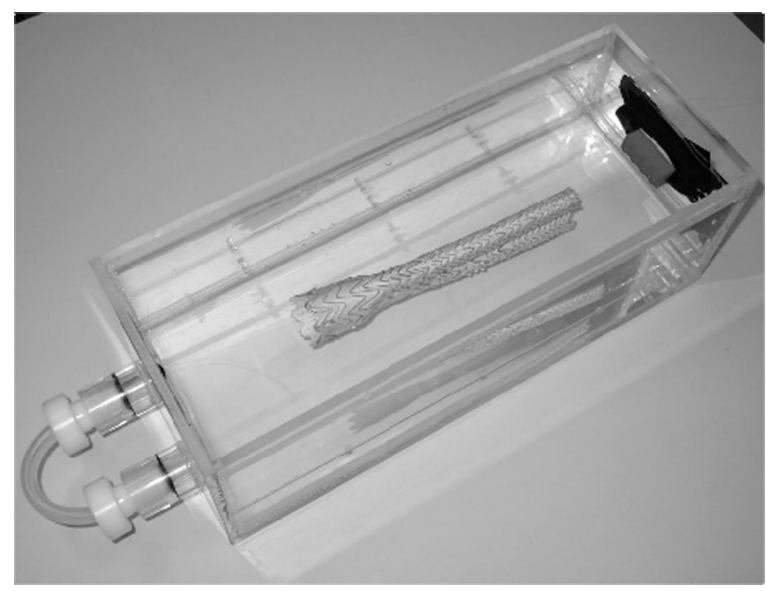

Figure $1 \diamond$ The endografts used in this study were tested in a water bath containing gadolinium. endografts were scanned: AneuRx (Medtronic Vascular, Santa Rosa, CA, USA), Lifepath (Baxter, Morton Grove, IL, USA), Talent (Medtronic Vascular), Excluder (Gore, Flagstaff, AZ, USA), Zenith (Cook, Bloomington, IN, USA), Quantum LP (now Fortron; Cordis, Warren, NJ, USA), and Ancure (Guidant, Menlo Park, CA, USA). The specific metal components of each graft are shown in Table 1. The grafts were mounted in a plastic container (Fig. 1) so that they hung freely in water containing an aqueous gadolinium-diethylenetriamine penta-acetic acid (GD-DPTA) solution that mimicked the relaxation properties of contrast-enhanced blood. The model was placed inside a birdcage head receiver coil. Scans were made on a clinical 1.5-T Gyroscan Intera MRI scanner (Philips Medical Systems, Best, The Netherlands).

\section{Data Acquisition}

Two MRI scan techniques were selected from among our clinical endograft surveillance protocols and slightly adapted owing to the smaller area of interest compared to the clinical setting. In our experience, standard gradient echo and spin echo scans provide sufficient information to evaluate the amount of radiofrequency (RF) shielding and the severity of susceptibility artifacts for all stentgrafts. Because of the capricious dependencies of the RF shielding phenomena on various stent and sequence-related factors, ${ }^{6}$ quantification of RF shielding is useful only when compared between grafts. The scan parameters relevant for artifact formation (echo time, read-out bandwidth, fold-over direction, 

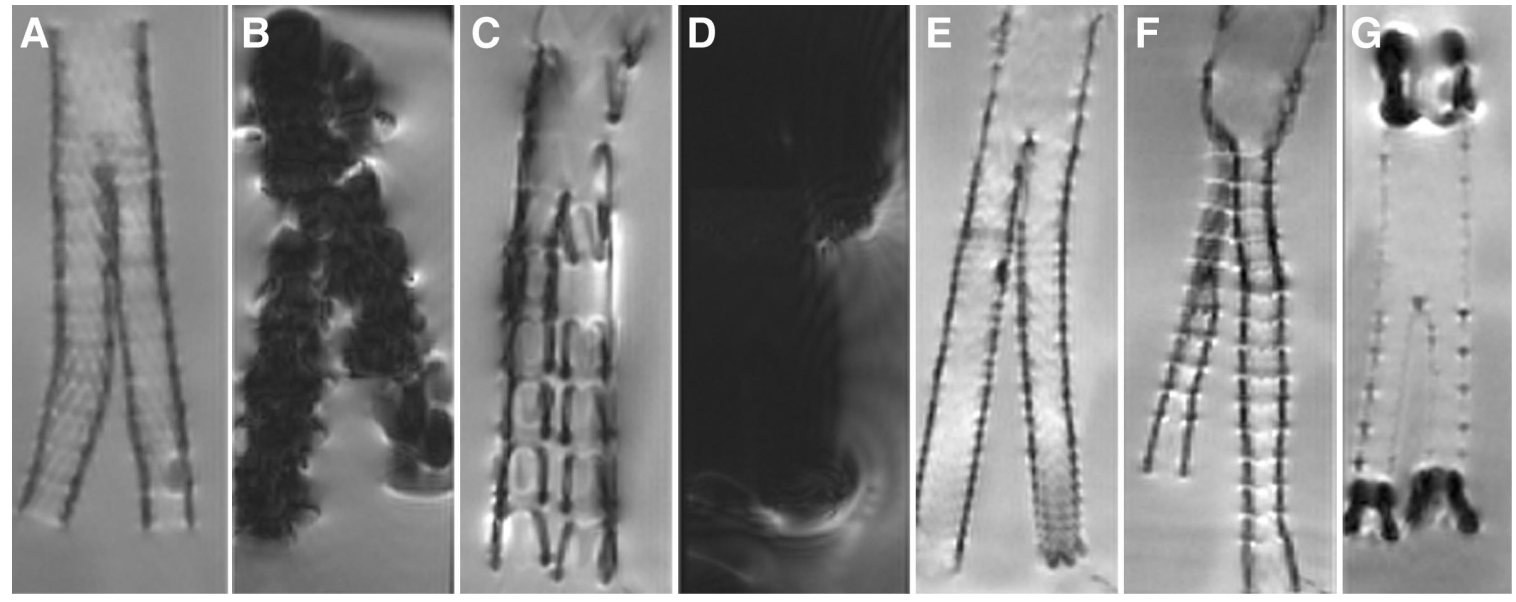

Figure 2 Single coronal slices taken from the 3-dimensional $T_{1}$-weighted spoiled gradient echo scan: (A) AneuRx, (B) Lifepath, (C) Talent, (D) Zenith, (E) Excluder, (F) Quantum LP, and (G) Ancure.

resolution) were chosen so that the resulting images were a representation of the impact of the artifacts on image quality and the diagnostic value of the scans in our MRI-based follow-up program.

Following a multistack survey, a 3-dimensional (3D) $T_{1}$-weighted spoiled gradient echo scan was obtained with a repetition time (TR) of $7.7 \mathrm{~ms}$, an echo time (TE) of $2.1 \mathrm{~ms}$, a flip angle $(\alpha)$ of $30^{\circ}$, a $4.0-\mathrm{mm}$ slice thickness with 2-mm reconstruction, a $256 \times 256 \mathrm{~mm}^{2}$ field of view (FOV), and a $256 \times 256$ acquisition matrix. A transverse $T_{1}$-weighted spin echo scan normally used for aneurysm volume measurements, endoleak detection, and investigation of thrombus organization was performed at

TABLE 2

Signal Attenuation Caused by the Endografts

\begin{tabular}{lcc}
\hline & Lumen & $\begin{array}{c}\text { Attachment } \\
\text { Sites }\end{array}$ \\
\hline AneuRx & $34 \% \pm 8 \%$ & $25 \% \pm 7 \%$ \\
Lifepath & NA & NA \\
Talent & $7 \% \pm 4 \%$ & $2 \% \pm 6 \%$ \\
Excluder & $8 \% \pm 4 \%$ & $15 \% \pm 8 \%$ \\
Zenith & NA & NA \\
Quantum LP & $71 \% \pm 5 \%$ & $66 \% \pm 8 \%$ \\
Ancure & $3 \% \pm 11 \%$ & $15 \% \pm 3 \%$ \\
&
\end{tabular}

Percentage attenuation is given as the mean \pm standard deviation.

NA: not available due to severe artifact; signal attenuations could not be quantified.
$\mathrm{TR} / \mathrm{TE} / \alpha=580 \mathrm{~ms} / 14 \mathrm{~ms} / 90^{\circ}, 3.0-\mathrm{mm}$ slice thickness, a $256 \times 256 \mathrm{~mm}^{2} \mathrm{FOV}$, and an acquisition matrix of $256 \times 256$.

\section{Scan Analysis}

The assessment of RF caging was expressed as a percentage of signal loss on the $\mathrm{T}_{1}$-weighted spin echo scan with respect to the undisturbed signal in the water/Gd-DTPA solution outside the endograft. Signal loss due to dephasing was assessed from the minimum intensity projections ( $\mathrm{mIP}$ ) of the $3 \mathrm{D} \mathrm{T}_{1^{-}}$ weighted spoiled gradient echo scans.

\section{RESULTS}

Imaging results of the endograft lumen on the 3D $\mathrm{T}_{1}$-weighted spoiled gradient echo scan (Fig. 2) showed that the Lifepath and Zenith devices had significant artifacts that may seriously affect the image quality of the lumen (Table 2). In the Ancure graft, depiction of the lumen was compromised at the attachment sites. The 4 other devices did not produce disturbing susceptibility artifacts. Slices of the $\mathrm{T}_{1}$-weighted spin echo scan through the body of the endograft (Fig. 3) demonstrated the differences in RF shielding among the different endografts.

The mIPs derived from the $3 \mathrm{D} \mathrm{T}_{1}$-weighted spoiled gradient echo scans (Fig. 4) demon- 

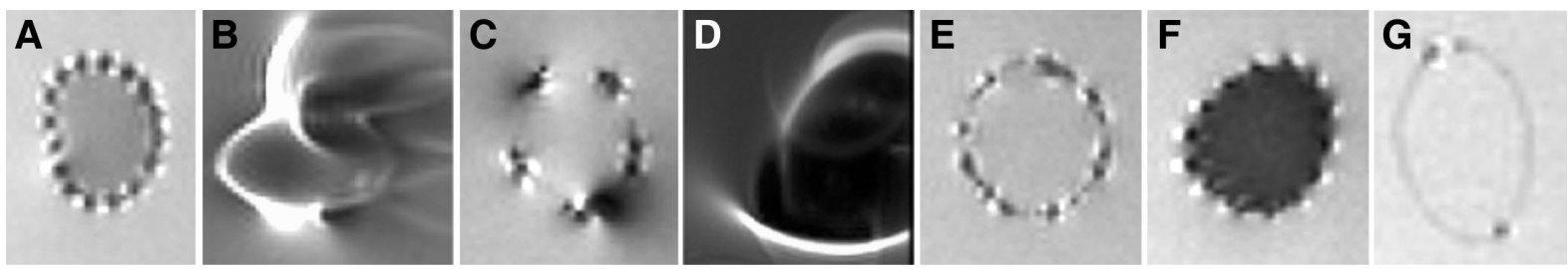

Figure $3 \triangleleft$ Cross-sections of the 7 endografts from the spin echo scan through the body of the stent-graft: (A) AneuRx, (B) Lifepath, (C) Talent, (D) Zenith, (E) Excluder, (F) Quantum LP, and $(\mathbf{G})$ Ancure. Note the radiofrequency caging artifacts, especially in $A$ and $F$, and the marker-induced artifacts on both sides on the Ancure graft (G).

strated that artifacts from nitinol-supported endografts do not compromise the depiction of the tissue directly surrounding the endograft (Table 3). Elgiloy and stainless steel skeletons, on the other hand, produce large artifacts, severely affecting the scan's diagnostic value.

\section{DISCUSSION}

This study evaluated the suitability of different endografts for an MRI-based endograft surveillance program. For an endograft to be MRI compatible, the artifacts that it produces should not degrade the diagnostic value of the images. Moreover, the implant may not incur safety problems while inside or in the vicinity of the scanner. In our model, we tried to emulate the in vivo situation as much as possible, although some factors that influence image quality clinically, such as respiratory motion, could not be duplicated. None- theless, our experiments allowed easy comparison of the various grafts with regard to different image artifacts.

Among the safety issues relating to the presence of metal in the strong magnetic field of an MRI scanner, ${ }^{7}$ the most obvious risk is dislodgement of the endograft by longitudinal forces and torques experienced by the ferromagnetic stent parts. The maximum torque will be experienced in the region of the highest magnetic field strength. The attractive force will be maximal where the product of the spatial gradient and the field strength is largest, which for most scanners is near the entrance of the bore. In our in vitro model, forces and torque were not observed, except for the Zenith and Lifepath devices. Whether the force exerted on these implants influences MRI safety cannot be concluded from this study. MRI safety of these endografts is hardly relevant for follow-up, though, since the large image artifacts make them MRI incom-
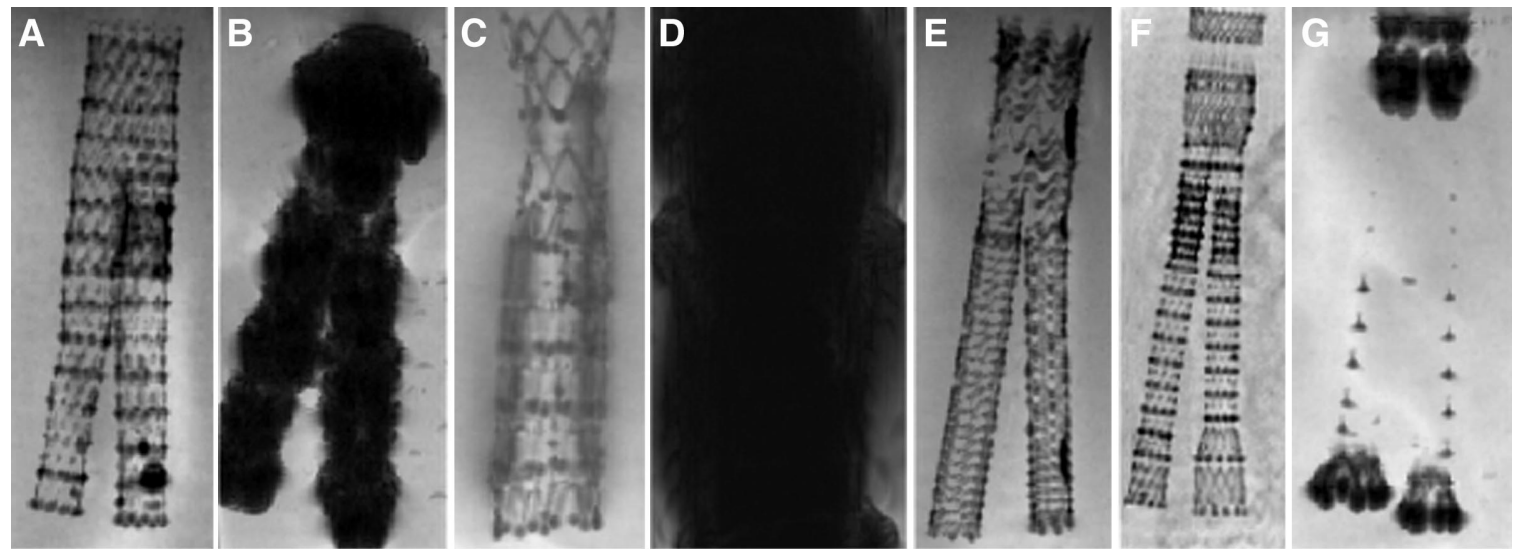

Figure 4 Minimum intensity projections from the spoiled gradient echo scans of the 7 endografts: (A) AneuRx, (B) Lifepath, (C) Talent, (D) Zenith, (E) Excluder, (F) Quantum LP, and (G) Ancure. 


\begin{tabular}{lll}
\hline & $\begin{array}{c}\text { TABLE 3 } \\
\text { Endograft Influence on Visualizing } \\
\text { Adjacent Structures }\end{array}$ \\
\hline & Body/Limbs & $\begin{array}{c}\text { Attachment } \\
\text { Sites }\end{array}$ \\
\hline AneuRx & No & No \\
Lifepath & Severe & Severe \\
Talent & Minor & Minor \\
Excluder & No & No \\
Zenith & Severe & Severe \\
Quantum LP & No & No \\
Ancure & No & Moderate \\
&
\end{tabular}

The attachment sites and the body/limbs were addressed separately.

patible and render MRI-based follow-up useless. $^{7}$

Another potential safety hazard associated with MRI examination of metallic implants is local heating of tissue surrounding the implant. Theoretically, 3 distinct processes could cause heating: resistive dissipation of energy from eddy currents, heating caused by currents induced in conducting loops, and heating by resonating RF waves. Eddy currents are present in every conducting material exposed to RF radiation. Induction currents, which may occur in loops from conductive materials, depend on the dimensions of the loop and the polarization of the RF pulse. Thermal energy deposits from these 2 processes have been studied in many metallic implants; rarely did the temperature increase exceed more then a few degrees Celsius. ${ }^{8-11}$ Therefore, these processes do not appear to be a substantial hazard in small metallic implants.

Resonating RF waves, however, have been shown to give a fast and significant increase in temperature. Elongated conducting structures, such as leads of active implants (e.g., pacemakers or deep brain stimulators) or metallic guidewires used during MRI-guided interventions, might act as linear antennas, causing excessive heating near the wire tips if standing waves are generated. ${ }^{8}$ This form of heating is therefore hazardous. These resonance effects, however, occur only when the length of the wire-like structure is over one half of the RF wavelength. With clinical 1.5-T scanners and human tissue, this is $\sim 26 \mathrm{~cm}^{8}$
For implants that are small compared to the RF wavelength used for excitation (e.g., stents, coils, clips, and the metallic parts in endografts), electromagnetic resonance is considered impossible. All endografts used in this study were under $18 \mathrm{~cm}$ in length. Furthermore, most endografts are composed of separate supporting stents not connected to each other. Consequently, no significant heating of these devices is expected. Accordingly, we have not carried out any experiments with regard to heating of tissue surrounding the endografts.

In general, there are 3 metal components of endografts that can cause artifacts in MRI scans: the supporting stent (if present), the metal attachment, and the metal markers used for fluoroscopic guidance during implantation. Basically, 2 types of metal-induced artifacts can occur. ${ }^{6}$ Susceptibility artifacts are caused by local differences in magnetic volume susceptibility $(\chi)$ between the metal in the implant and the surrounding tissues. These differences lead to local inhomogeneities in the main magnetic field, which cause geometric distortions and local signal loss around the metallic parts due to dephasing of the nuclear spins. The severity of these artifacts is dependent on the magnetic susceptibility of the metallic materials. As we saw with the Zenith and Lifepath models, the presence of ferromagnetic materials (with $\chi>10^{-2}$ ) in endografts can cause overwhelming susceptibility artifacts that make evaluation of the abdomen impossible. ${ }^{12}$

In addition, in supported endografts with a cage-like stent structure, depiction of the lumen can be compromised by RF shielding of the implant's lumen. Especially in implants made of low-artifact materials, in which susceptibility artifacts are small, RF-related artifacts may play an important role. The geometry and orientation of the conducting loops in the endograft with respect to the polarization of the RF magnetic field and the receiver/ transmitter fields of the coils used for MRI imaging are important. The extent to which RF caging occurs is therefore strongly dependent on the geometrical orientation of the stents and the conductance of the material from which the stent is made. ${ }^{13}$

All fully supported grafts showed some RF 
caging artifacts, reducing the lumen signal. The unsupported Ancure device did not show an appreciable signal loss of the graft interior. If desired, these RF artifacts can be diminished by increasing the RF power of the excitation pulse, thereby improving lumen visualization. ${ }^{13}$ In the Ancure graft, however, depiction of the lumen at the level of the attachment sites will stay insufficient because of the relatively large susceptibility artifacts caused by the cobalt-chromium alloy used in the attachment systems. From our clinical experience with the Ancure and Excluder, we do not believe that increasing the RF power is necessary, as the contrast-enhanced signal from the lumen is enough for diagnostic purposes. However, it must be kept in mind that the results of signal loss presented here are not absolute values. The amount of caging will be dependent on the position of the endograft in the scanner and on the flip angle.

In conclusion, all endografts in our study showed metal-induced artifacts to some extent, but most did not influence the appreciation of the graft's exterior or interior. All fully supported endografts showed some amount of signal loss due to RF caging. For the Ancure graft, MRI-based follow-up is not impossible, but the attachment sites do pose problems. An MRI-based follow-up of the Lifepath and the Zenith will be useless, as the images will have no diagnostic value. These grafts are better assessed by CTA. All nitinol stents appear to be MRI compatible. In our experience, the Talent, AneuRx, Quantum LP, and the Excluder are well suited for a surveillance program that features MRI-based imaging techniques.

\section{REFERENCES}

1. Cejna M, Loewe $C$, Schoder $M$, et al. MR angiography vs CT angiography in the follow-up of nitinol stent grafts in endoluminally treated aortic aneurysms. Eur Radiol. 2002;12:24432450.
2. Haulon S, Lions $C$, McFadden EP, et al. Prospective evaluation of magnetic resonance imaging after endovascular treatment of infrarenal aortic aneurysms. Eur J Vasc Endovasc Surg. 2001;22:62-69.

3. Haulon S, Willoteaux S, Koussa M, et al. Diagnosis and treatment of type II endoleak after stent placement for exclusion of an abdominal aortic aneurysm. Ann Vasc Surg. 2001;15:148154.

4. Jacobs TS, Won J, Gravereaux RC, et al. Mechanical failure of prosthetic human implants: a 10-year experience with aortic stent graft devices. J Vasc Surg. 2003;37:16-26.

5. Mita T, Arita T, Matsunaga N, et al. Complications of endovascular repair of thoracic and abdominal aortic aneurysm: an imaging spectrum. Radiographics. 2000;20:1263-1278.

6. Bartels LW, Smits HF, Bakker CJ, et al. MR imaging of vascular stents: effects of susceptibility, flow, and radiofrequency eddy currents. $J$ Vasc Interv Radiol. 2001;12:365-371.

7. Shellock FG. Biomedical implants and devices: assessment of magnetic field interactions with a 3.0-Tesla MR system. J Magn Reson Imaging. 2002;16:721-732.

8. Konings MK, Bartels LW, Smits HF, et ak. Heating around intravascular guidewires by resonating RF waves. J Magn Reson Imaging. 2000; 12:79-85.

9. Buchli R, Boesiger P, Meier D. Heating effects of metallic implants by MRI examinations. Magn Reson Med. 1988;7:255-261.

10. Davis PL, Crooks L, Arakawa M, et al. Potential hazards in NMR imaging: heating effects of changing magnetic fields and RF fields on small metallic implants. AJR Am J Roentgenol. 1981;137:857-860.

11. Shellock FG, Curtis JS. MR imaging and biomedical implants, materials, and devices: an updated review. Radiology. 1991;180:541-550.

12. Merkle EM, Klein S, Kramer SC, et al. MR angiographic findings in patients with aortic endoprostheses. AJR Am J Roentgenol. 2002; 178:641-648.

13. Bartels LW, Bakker CJ, Viergever MA. Improved lumen visualization in metallic vascular implants by reducing RF artifacts. Magn Reson Med. 2002;47:171-180. 